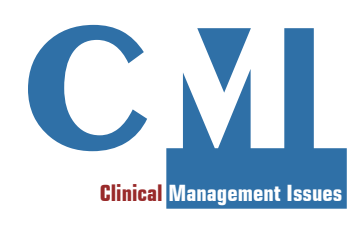

\title{
Life-threatening Cerebral Venous Thrombosis: a case report
}

\begin{abstract}
Cerebral venous sinus thrombosis (CVT) can compromise dural sinus, cerebral veins or both. It is an uncommon condition and it is more prevalent in young women. Several prothrombotic states are the principal predisposing factors. Clinical spectrum of presentation is wide, so this entity requires a high suspect index for correct and prompt diagnosis. CVT may develop serious complications that can be life-threatening such as hemorrhagic venous infarctions, cerebral edema, and intracranial hypertension.

This report describes the case of a woman who was in treatment for unspecific vaginal bleeding with oral contraceptives. Suddenly she deteriorated to coma with severe respiratory compromise. Neuroimaging showed thrombosis of multiple venous sinus. Physiological neuroprotection, osmotherapy, mechanical ventilation and anticoagulation therapy were the keystones of treatment. In a few months, the patient has recovered a good functional status, while maintaining a motor deficit on the right hand.
\end{abstract}

Keywords: Cerebral venous thrombosis; Hemorrhagic infarctions; Intracranial hypertension; Magnetic resonance image; Anticoagulation

CMI 2016; 10(2): 49-53

http://dx.doi.org/10.7175/cmi.v10i1.1253

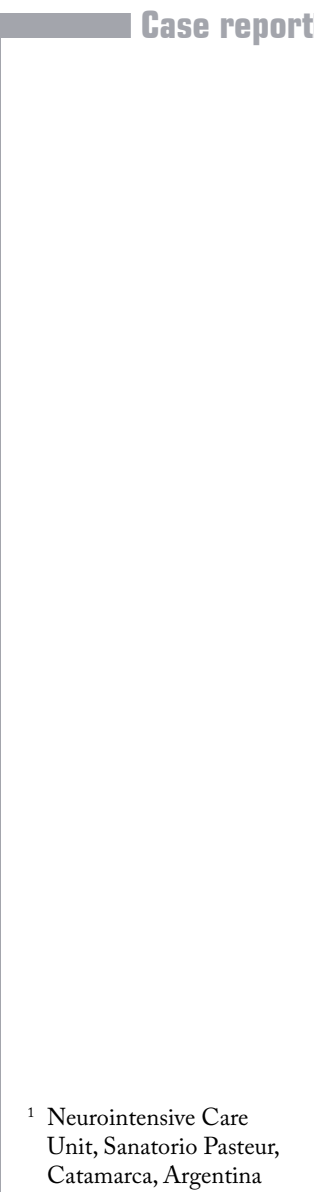

\section{INTRODUCTION}

Cerebral Venous Thrombosis (CVT) represents $0.5 \%$ of all strokes with an estimated prevalence of 5 people in 1 million each year. CVT may include thrombosis of cerebral veins and major dural sinuses [1-4]. This entity has a higher prevalence in young women during pregnancy, puerperium or taking oral contraceptives [1-4]. Thrombophilia, infections of maxillofacial region and malignances are other important predisposing factors [1]. Usually, CVT has a favorable prognosis, but it can be life-threatening in few cases [4]. The pathophysiological substrates for central nervous system dysfunction are cerebral edema, venous infarctions and intracranial hypertension [1-5]. Magnetic Resonance Imaging (MRI) is the keystone for the diagnosis and follow-up [1-
Why do we describe this case?

CVT is a rare but potentially lifethreatening type of stroke that occurs principally in young women. From a pathophysiological point of view, the thrombosis of the cerebral venous system increase microvascular pressure and blood brain barrier permeability, and compromise cerebrospinal fluid $a b$ sorption. Cerebral edema, parenchymal bemorrhages, acute hydrocephalus and intracranial bypertension are substrates for clinical presentation. Different syndromes are classically described; intracranial hypertension (headache), focal deficits (paresis, aphasia), seizures and encephalopathy.
Corresponding author Daniel Agustín Godoy Neurointensive Care Unit, Sanatorio Pasteur, Chacabuco 675, 4700 Catamarca, Argentina dagodoytorres@yahoo.com.ar

Disclosure

The authors declare they have no competing financial interests concerning the topics of this article 
Figure 1. Neuroimaging at admission.

(A) CT scan shows bilateral occipitoparietal infarctions with hemorrhagic transformation and peri-lesional edema; (B) MRI in Axial T2-weighted image shows increased signal intensity in both occipital and left frontal lobes; (C) MRI venography demonstrating occlusion of superior and inferior sagittal (arrows) and (D) left transverse sinus (arrow)
4]. Anticoagulation therapy, neurophysiological protection [6] and osmotherapy are the mainstays for acute management [1-6].

\section{CASE REPORT}

A 54-year-old woman is admitted to the emergency department in a coma, with a decerebration rigidity, no pupillary alterations, and an irregular breathing pattern. Her score on the Glasgow Coma Scale was 4. Vital parameters were: mean arterial pressure (MAP) $88 \mathrm{mmHg}$, heart rate 126 beats/min (sinusal rhythm), central temperature $36.3^{\circ} \mathrm{C}$ (esophagus), and respiratory rate 11 breaths/min. Three days before the patient had suffered from a headache and progressive left hemiparesis. On admission, the patient was taking enalapril arterial hypertension, and oral and intravenous contraceptives, started 3 months earlier due to non-specific vaginal bleeding.

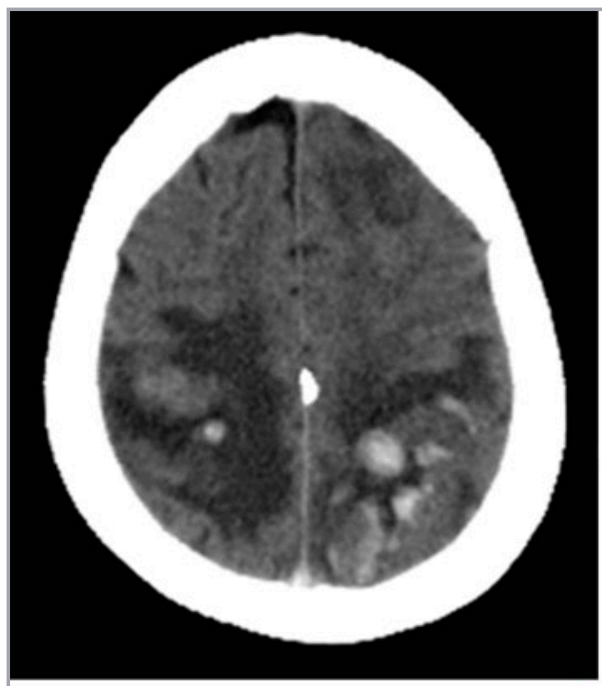

A

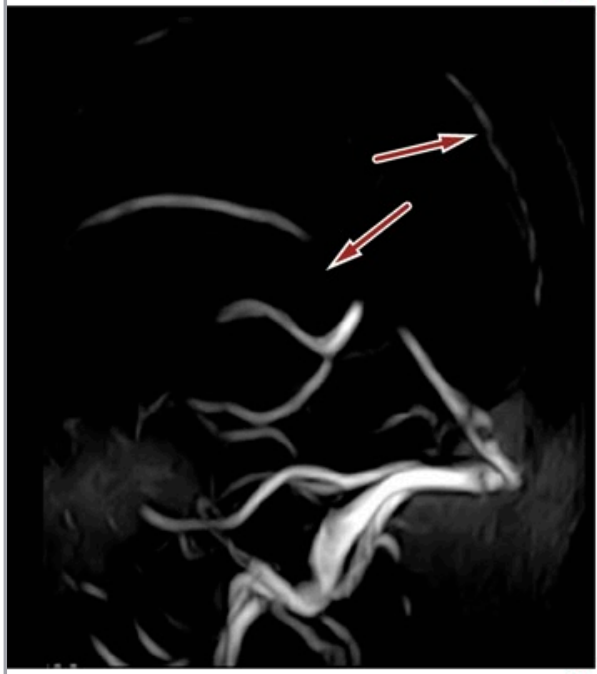

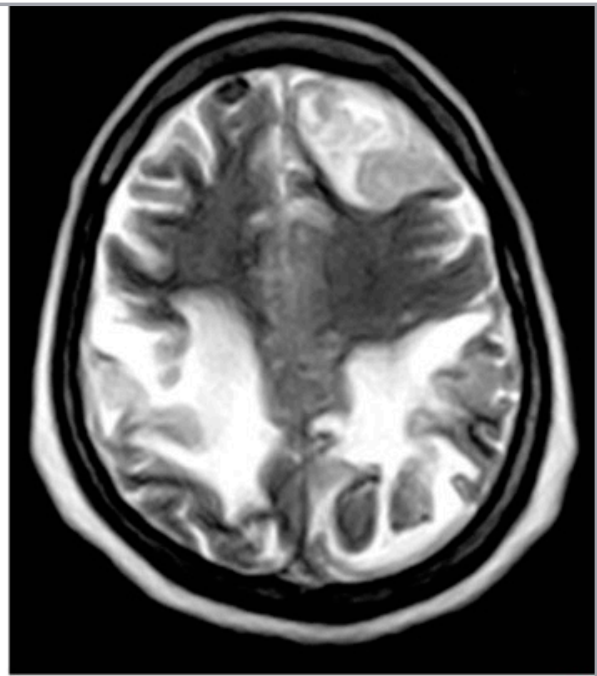

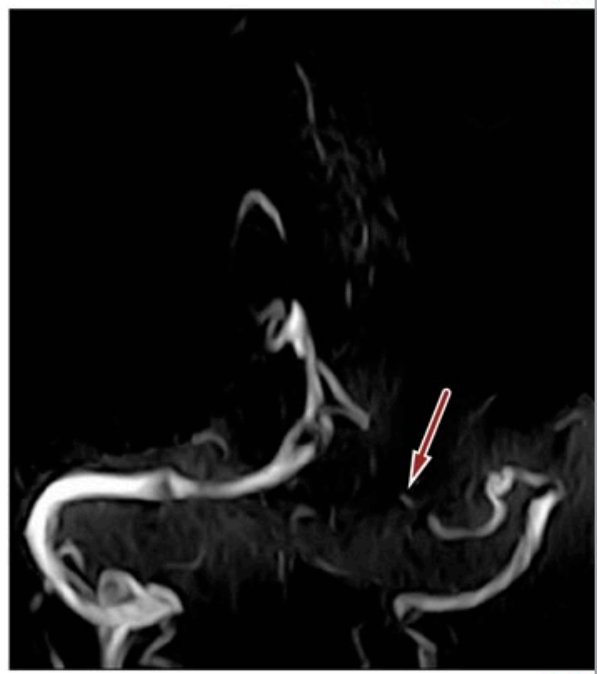

After an adequate and complete resuscitation, including control of upper airways with orotracheal intubation, oxygenation, and hemodynamic stability, she was transferred to the radiology department.

An unenhanced computed tomography (CT) scan in sagittal view showed spontaneously hyperintense superior longitudinal sinus, and bilateral occipital hemorrhagic infarctions. MRI showed hemorrhagic infarctions and occlusion of several sinuses. (Figure 1). Electroencephalogram showed non-convulsive status epilepticus.

After the diagnostic imaging, the patient was transferred to the neurointensive care unit. She was sedated (midazolam 3-5 mg/ $\mathrm{kg} / \mathrm{h}$ ), anesthetized (remifentanil 0.25-0.4 $\mathrm{mcg} / \mathrm{kg} / \mathrm{min}$ ) and mechanically ventilated. Then she was treated with physiologic neuroprotection [5]5 and osmotherapy. At the same time an anticoagulation therapy with low weight molecular heparin (eno- 


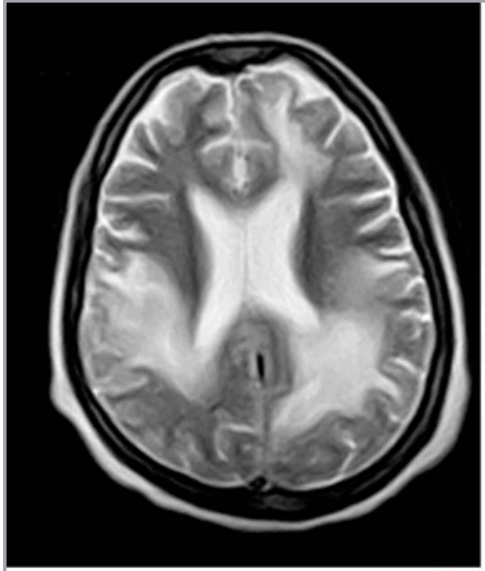

A

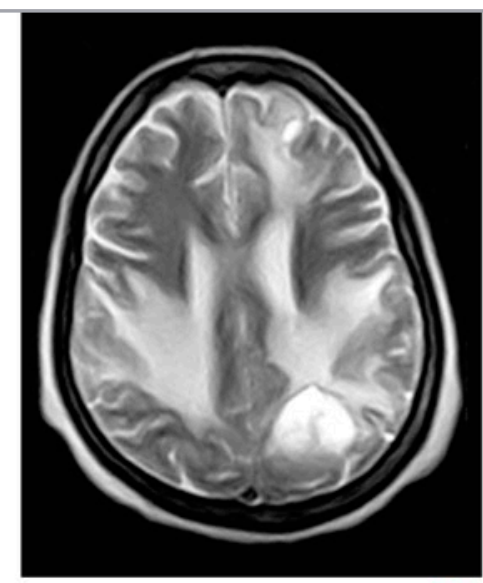

B

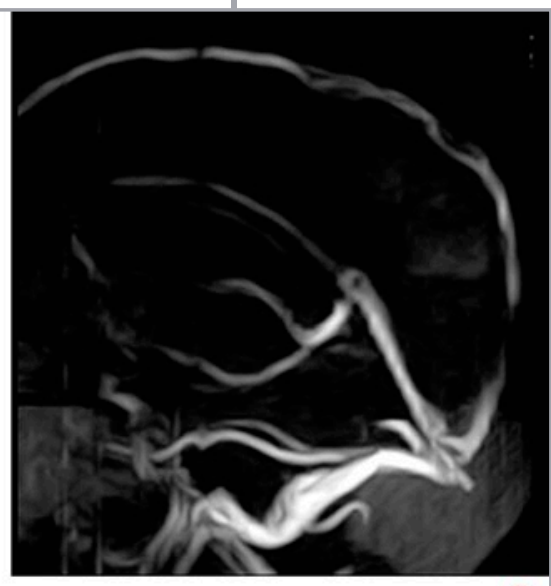

xaparin $1 \mathrm{mg} / \mathrm{kg}$ twice daily) and warfarin $(5 \mathrm{mg} /$ day) was started in order to obtain a targeted international normalized ratio (INR) of 2.5-3.

An exhaustive search of predisposing factors ruled out cancer and infections of the ear, paranasal sinuses, mouth or face. Prothrombotic predisposing causes of CVT (antiphospholipid syndrome, deficit antithrombin III, protein C and S) were excluded. Fibrinogen and VIII factor levels were in the normal range, and no mutation of Leiden factor was found. The non-convulsive status epilepticus was controlled with midazolam $(5 \mathrm{mg} / \mathrm{h})$ and levetiracetam $(3 \mathrm{~g} /$ day $)$.

After two weeks of mechanical ventilation, a tracheostomy was performed. Atelectasis and ventilator-associated pneumonia were corrected with physiotherapy and antibiotics (imipenem $2 \mathrm{~g} /$ day and amikacin $1 \mathrm{~g} /$ day). A control MRI showed the recanalization of affected sinuses (Figure 2).

After 31 days, the patient was discharged to rehabilitation unit. She appeared lucid, oriented and in a good consciousness state but she has a severe paresis of her left arm and leg. After two months of rehabilitation, neurocognitive functions have shown a strong recovery, even though her right hand still showed difficulty moving.

\section{DISCUSSION}

CVT is an entity with pathophysiological, diagnostic and therapeutic features that differ from stroke of arterial origin [1-4]. The clinical spectrum of presentation is highly variable and can be confused with other entities [1-4]. The onset of symptoms may be acute, subacute or chronic, while signs and symptoms depend primarily on the location and extent of thrombosis, as well as the pathophysiology of venous occlusion is characterized by the development of cerebral edema, venous infarcts and intracranial hypertension due to mass effect or hydrocephalus [1-4]. Unspecific headaches, without characteristic features that clearly distinguish them from others, are present in over $90 \%$ of cases. It is therefore extremely important to take in mind this entity, in order to avoid delays in diagnosis, especially in young women under contraceptives oral or hormonal therapy or during pregnancy and postpartum [1-4].

In $40 \%$ of cases, the presenting symptom is a focal deficit, motor (hemiparesis-plegia), sensory or language disorders, while in more than one $1 / 3$ of cases the onset of thrombosis is indicated by the presence of seizures [1-4]. Mental status changes dominate the clinical
Figure 2. Follow up MRI and MRI venography that showed evolution of venous infarctions $(A, B)$ and recanalization of superior and inferior sagittal sinuses $(C)$

What should the clinician ask him/herself or the patient?

While CVT has different presentation forms, in over $90 \%$ of cases the predominant symptom is a headache. Headaches are a very common cause of consultation, especially in young women. CVT is a diagnosis to keep in mind! CVT requires a bigh index of suspicion and meticulous research of predisposing factors (pregnancy, the use of oral contraceptives, maxillofacial infections, malignancies, thrombophilia), because it is a treatable entity with good prognosis if early diagnosis and appropriate treatment are undertaken. 


\begin{tabular}{|c|c|}
\hline Women's specific & $\begin{array}{l}\text { - Pregnancy } \\
\text { - Puerperium } \\
\text { - Oral contraceptives } \\
\text { - Hormonal therapy }\end{array}$ \\
\hline Thrombofilic states & $\begin{array}{l}\text { - Antiphospholipid syndrome } \\
\text { - Deficiencies of antithrombin, protein C and S } \\
\text { - Factor Leiden (V) mutation } \\
\text { - Prothrombin gene mutation } \\
\text { - Hyperhomocysteinemia } \\
\text { - Nephrotic syndrome }\end{array}$ \\
\hline Infections & $\begin{array}{l}\text { - Otitis, mastoiditis, sinusitis } \\
\text { - Meningitis } \\
\text { - Systemic infections }\end{array}$ \\
\hline $\begin{array}{l}\text { Chronic Inflammatory } \\
\text { Diseases }\end{array}$ & $\begin{array}{l}\text { - Systemic erithematosous lupus } \\
\text { - Vasculitides } \\
\text { - Inflammatory bowel disease }\end{array}$ \\
\hline Cancer & - All types \\
\hline Hematologic disorders & $\begin{array}{l}\text { - Polycitemia } \\
\text { - Essential thrombocytosis } \\
\text { - Paroxysmal nocturnal hemoglobinuria }\end{array}$ \\
\hline Trauma & $\begin{array}{l}\text { - Traumatic brain injury } \\
\text { - Maxillo-facial trauma } \\
\text { - Jugular venous catheterization } \\
\text { - Neurosurgery } \\
\text { - Lumbar puncture }\end{array}$ \\
\hline
\end{tabular}

Table I. Predisposing factors for $C V T$ development
- control of seizures, and intracranial hypertension;

- anticoagulation;

- monitoring of complications that may require rescue therapies.

The best way to avoid secondary insults is through physiological homeostasis or neuroprotection [6]; that is, simply, to maintain basic physiological variables within normal ranges, ensuring ventilation, adequate oxygenation, and hemodynamic stability [6] Additionally, a strict control of temperature, blood glucose and serum sodium is very important [6]. Seizure control is no different from other circumstances, only we emphasize the value of EEG monitoring without which it is impossible to detect nonconvulsive states, principally in individuals under mechanical ventilation, sedation and analgesia [1-5].

Anticoagulation is the key to the therapy because it prevents the thrombus propagation, helps the venous recanalization of occluded territories, and prevents potentially lethal complications, such as deep vein thrombosis and pulmonary embolism [1-5]. Despite the controversy regarding anticoagulation in individuals with bleeding or venous infarctions and based on randomized controlled trials, current guidelines recommend starting immediate anticoagulation to achieve an INR value between 2 and 3 [2]. Unfractionated heparin or low molecular weight heparin are safe and effective options, and there is no evidence to support the use of one rather than the other [1-5].

Generally, the above measures are sufficient to control intracranial hypertension; occasionally osmotherapy (hypertonic saline solutions or mannitol) or external ventricular drainage may be needed [5]. While most individuals respond to the therapeutic measures mentioned above, some require additional procedures such as direct venous fibrinolysis through catheter by endovascular therapy and/or decompressive craniectomy for control of refractory intracranial hypertension [1-5].

Despite the favorable prognosis usually associated with this disease, the case presented here summarizes all aspects of the management of a potentially lethal clinical form, that can occur in approximately one of every four cases of CVT. To avoid diagnostic and therapeutic delays, it is essential to consider this entity and establish a high index of suspicion for individuals with a headache, focal neurologic deficits or mental status changes, 
especially when one or more predisposing factors are present. In these cases, a systematic pathway to study and confirm the original etiology should trigger. Clinical suspicion of CVT requires confirmation with diagnostic imaging, preferably MRI. Anticoagulation therapy is mandatory, despite the presence of cerebral hemorrhages.
Key points

- CVT is a neurologic emergency that require high suspicion index

- Screening of predisposing factors is mandatory

- MRI is a gold standard for diagnosis and follow up

- Anticoagulation is the key stone of the treatment

- In certain situations CVT can be life threatening

\section{REFERENCES}

1. Bushell C, Saposnik G. Evaluation and management of cerebral venous thrombosis. Continuum 2014; 20: 335-351. doi: 10.1212/01.CON.0000446105.67173.a8

2. Saposnik G, Barinagarrementeria F, Brown R, et al. Diagnosis and management of cerebral venous thrombosis: a statement for healthcare professionals from the American Heart Association/American Stroke Association. Stroke 2011; 42: 1158-1192. doi: 10.1161/ STR.0b013e31820a8364.

3. Piazza G. Cerebral Venous Thrombosis. Circulation 2012; 125: 1704-1709. doi: 10.1161/ CIRCULATIONAHA.111.067835.

4. Ferro J, Canhao P, Stam J et al; ISCVT Investigators. Prognosis of cerebral vein and dural sinus thrombosis: results of the International Study on Cerebral Vein and Dural Sinus Thrombosis (ISCVT). Stroke 2004; 35: 664-670

5. Fam D, Saposnik G; Stroke Outcomes Research Canada Working Group. Critical care management of cerebral venous thrombosis. Curr Opin Crit Care 2016;22: 113-9. doi: 10.1097/ MCC. 0000000000000278

6. Godoy DA, Piñero GR, Koller P, et al. Steps to consider in the approach and management of critically ill patient with spontaneous intracerebral hemorrhage. World J Crit Care Med 2015; 4: 213-29. doi: 10.5492/wjccm.v4.i3.213. eCollection 2015

\section{PROPOSED ALGORITHM FOR CEREBRAL VENOUS THROMBOSIS (CVT) MANAGEMENT}

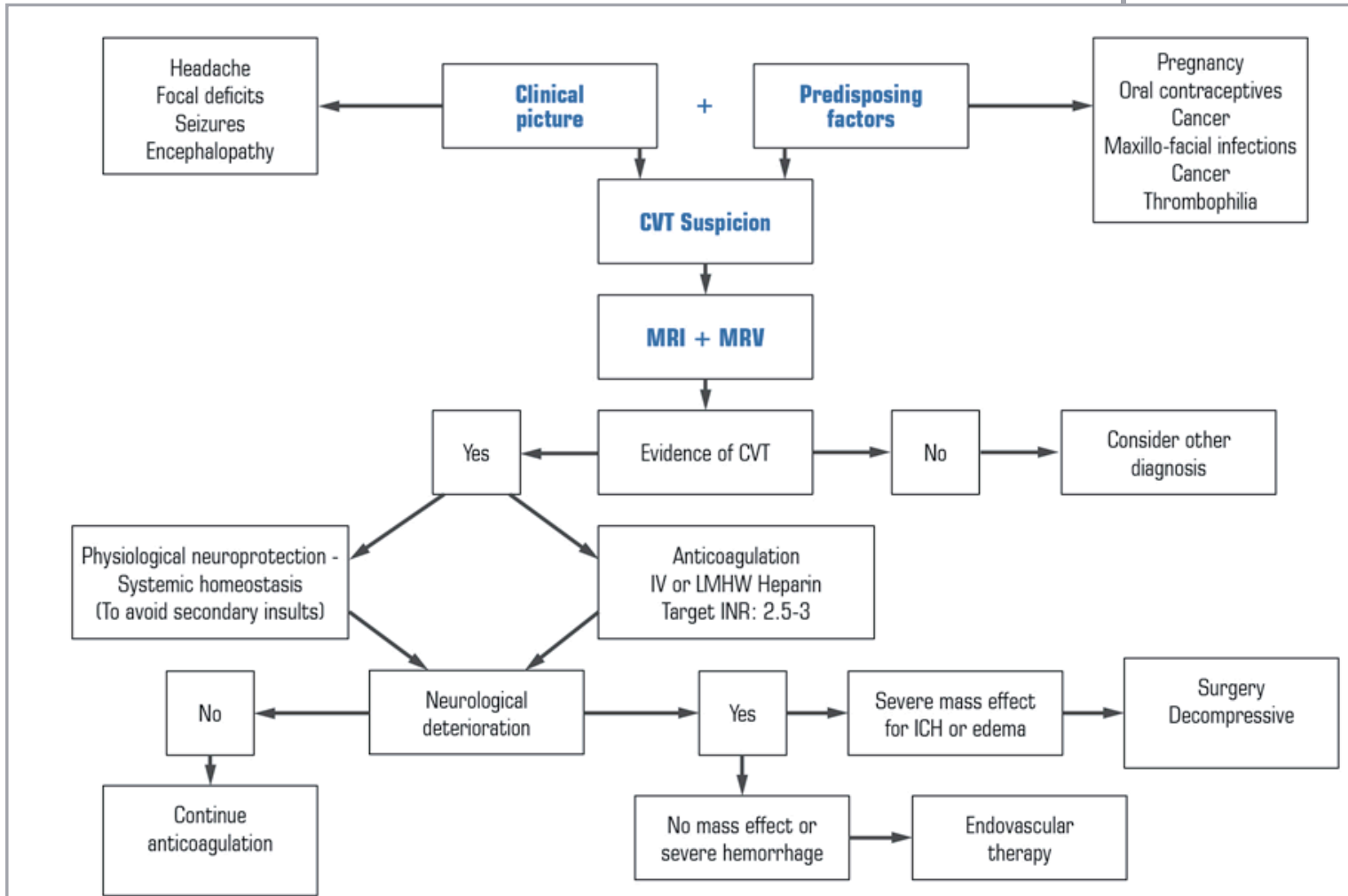

MRI = magnetic resonance image $\mathrm{MRV}=$ magnetic resonance venography; $\mathrm{IV}=$ intravenous;

LMHW = low molecular heparin weight; INR = international normalized ratio; ICH $=$ intracerebral hemorrhage 